\title{
The Dual Challenges for FIBXTEM in the Era of sub-50nm Feature Sizes
}

\author{
B. Tracy*, A. Myers*, M. Sidorov*, A. delRosario*, E. Adem*, B. Phillips, J. Gray, and M.
} Guillena*

*Materials Characterization Lab, Spansion Sunnyvale, Ca

Since the first demonstration of the usefulness of FIB technology for preparing site-specific TEM cross sections from sub-micron features (1), the focused ion beam has now become the method of choice for TEM sample preparation in materials science. The advantages of this method have been reviewed extensively elsewhere (2), but essentially, it is now possible to prepare a TEM sample from almost any material that can be introduced into a FIB chamber. FIBXTEM is especially prevalent in the semiconductor (3) and hard drive industries (4).

However, in the era of sub-50nm feature sizes, two distinct TEM specimen preparation challenges have arisen: (1) the production of feature sizes significantly smaller than the normal thickness of TEM samples, and, (2) the choice of capping material during FIBXTEM preparation is now nearly as important as the material of the sample itself. It is the purpose of this paper to illustrate these two points and to present some of the solutions our laboratory has developed to mitigate their effects.

\section{Feature Sizes Smaller than Nominal TEM Sample Thickness}

The use of 193nm immersion lithography and the implementation of "clever spacer technologies" to define and produce sub 50nm feature sizes in the IC industry present the TEM analyst with the special challenge of producing TEM samples that contain only the feature of interest (FOI), without inclusion of any undesired layers lying in front of or behind the FOI.

Similarly, the field of Nanotechnology yields samples that are similarly size-constrained. It is now desirable to make a TEM cross section from a specific $20 \mathrm{~nm}$ nanotube (5). The method to produce such samples in an artifact-free manner is the challenge over the next five years.

There are several possible solutions available to cope with this new era of sub 50nm feature sizes. These include the use of highly accurate FIB methods, low energy FIB milling, electron tomography, chemical imaging and energy filtered imaging. Ultra high resolution SEM imaging looks especially attractive because it avoids both sample the projection and thickness problems. Please refer to Figure 1.

The development of $45 \mathrm{~nm}$ design rule trench isolations is especially challenging for TEM sample preparation. Cross sectioning normal to the long axis of the trench is fairly straightforward; however, cross sections in the opposite direction are very challenging as the FOI is on the order of $40 \mathrm{~nm}$ and is encased in the trench isolation oxide. Even when the specimen is perfectly made, there is precious little remaining silicon thickness available to generate image contrast. Please refer to Figure 2 which shows how the excellent secondary emission properties of thin oxides are used to judge the exact plane of the cross section. 


\section{The Choice of Capping Materials: Now more important than ever}

In order to shield the near surface of the sample from the harmful effects of the energetic ion beam, nearly all FIB-based sample prep methods employ a sputter resistant capping material. In our laboratory, we have experimented with at least nine different capping materials in order to maximize final image quality. Indeed the capping material may be critical to a successful outcome. We will illustrate just a few of these materials, including: SiN, Sharpie, Pt (ion and eBeam), carbon (ion and eBeam), sputtered carbon, epoxy, and Spin on Glass (SOG). Despite a great deal of effort $(6,7,8)$, there is a class of materials (soft polymers such as photo resist) for which there is no completely acceptable solution. The triple requirement of low heat input, no mechanical force, and no solvents has narrowed the possible capping choices to sputtered carbon with its less than perfect step coverage.

As an illustration of the importance of capping material, we present a TEM image of an IC defect detected during "Back End of Line" processing. In order to relocate this defect, the intact $300 \mathrm{~mm}$ wafer requires coordinate navigation in a whole wafer FIB. The defect is at the near surface, making conventional ion beam based platinum deposition unacceptable due to damage. Excellent results, Figure 3, were obtained using highly localized eBeam deposited carbon that not only protects the defect but provides high layer contrast.

\section{References}

[1] D. P. Basile, et al., MRS Specimen Preparation III, Vol. 254 (1992), 23.

[2] L. Giannuzzi, and F. Stevie, Springer, 2005.

[3] S. Nakahara, Elsevier Science, 2003.

[4] H. Wang, J. Fang, J. Arjavac, R. Kellner, MICROSCOPY TODAY, January 2008, 27.

[5] S. Aloni, Private Communication, Lawrence Berkeley Laboratory, 2009.

[6] B. Tracy, K. Alberi; and S. Tabrez, 92 July 2004 MICRO.

[7] J. Clarke, M. Schmidt, G. Orji, JVST,. B Vol. 25, Issue 6, pp. 2526-2530 (Nov 2007).

[8] B. Rijpers, D. Verkleij, E. Langer, ISTFA 33, Nov 4-8, 2007.

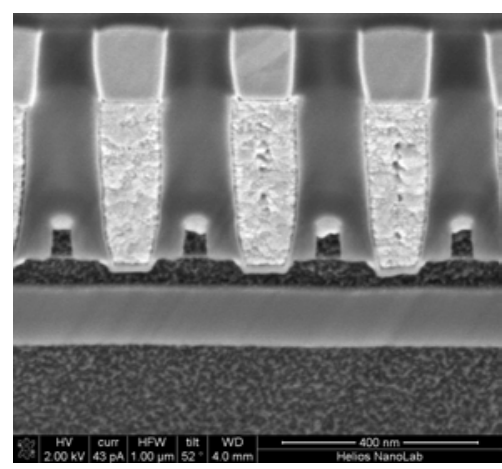

Fig 1, XSEM, great contrast

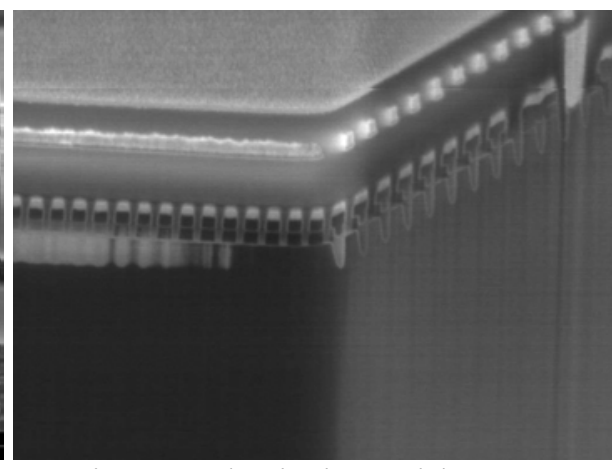

Fig 2, endpointing with SE's

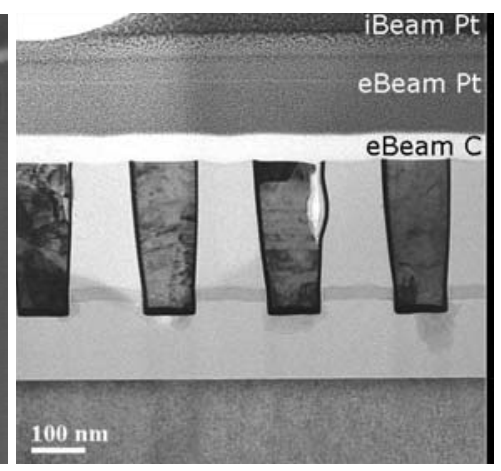

Fig 3, ebeam carbon cap 